\title{
HIV/TB Integrated Services: Perception of HIV and TB Clients in a Tertiary Health Facility in Lagos State Nigeria
}

\author{
Stellamaris Moronkeji ${ }^{1}$, Ebenezer Obi Daniel ${ }^{1, ~}$, Ogunnaike Adewale Adeyemi ${ }^{2}$, \\ Paul Olaiya Abiodun ${ }^{1}$, Lily Ize-Iyamu ${ }^{3}$, Abuworonye Fadeke ${ }^{4}$, Atabo Unekwu John ${ }^{5}$, \\ Olayinka Victor Ojo ${ }^{1}$, Israel Olukayode Popoola ${ }^{6}$ \\ ${ }^{1}$ Department of Public Health, School of Public Health, Texila American University, Georgetown Guyana, South America \\ ${ }^{2}$ Department of Health Policy and Management, Faculty of Public Health, University of Ibadan, Ibadan, Nigeria \\ ${ }^{3}$ Department of Medical Laboratory Sciences, Faculty of Basic Medical Sciences, University of Benin, Benin, Nigeria \\ ${ }^{4}$ Department of Health Studies, Faculty of Social Sciences, London Metropolitan University, London, United Kingdom \\ ${ }^{5}$ Department of History and International Studies, Faculty of Social Sciences, Nigerian Defence Academy, Kaduna, Nigeria \\ ${ }^{6}$ Department of Epidemiology and Community Health, Faculty of Medicine, University of Ilorin, Ilorin, Nigeria
}

\section{Email address:}

dannypressy@yahoo.com (E. O. Daniel)

${ }^{*}$ Corresponding author

\section{To cite this article:}

Stellamaris Moronkeji, Ebenezer Obi Daniel, Ogunnaike Adewale Adeyemi, Paul Olaiya Abiodun, Lily Ize-Iyamu, Abuworonye Fadeke, Atabo Unekwu John, Olayinka Victor Ojo, Israel Olukayode Popoola. HIV/TB Integrated Services: Perception of HIV and TB Clients in a Tertiary Health Facility in Lagos State Nigeria. Journal of Family Medicine and Health Care. Vol. 5, No. 3, 2019, pp. $32-37$.

doi: $10.11648 /$ j.jfmhc.20190503.12

Received: August 27, 2019; Accepted: September 9, 2019; Published: September 18, 2019

\begin{abstract}
HIV/AIDS remains a global public health issue which has claimed an estimated of 35 million lives since the start of the epidemic and further 78 million people still living with the virus. Tuberculosis (TB) is a major disease with high incident cases and over 1.3 million deaths recorded in 2012 even with the availability of effective chemotherapy. This survey is a descriptive, cross-sectional quantitative study among 87 enrolled clients attending HIV/TB integrated service at Lagos State University Teaching Hospital, (LASUTH) Lagos in Nigeria. Data collected were analyzed with SPSS version 21 with Chisquare used to test for association. Eighty percent (80) of the HIV clients were female while the TB patients were $41 \%$ male. Most of the respondents had only secondary school education and were married as at the time of the survey. Generally, unprotected sexual intercourse with a HIV infected person is the most known mode of spreading HIV among respondents. Majority of the respondents receives treatment for both TB and HIV in the same facility and view antiretrovirals as drugs responsible for preventing death from HIV/AIDS. While over $70 \%$ of the respondents agreed to like being visited by healthcare workers, over $60 \%$ reported to have never being visited by healthcare workers. The most reported advantages of integrated services for HIV and TB were "save cost for transportation and save time". The survey revealed good knowledge about HIV and TB among respondents while also showing that perception about integrated HIV and TB services is welcomed among the respondents. Increasing the number of healthcare workers and incentivizing the approach for healthcare workers were recommended.
\end{abstract}

Keywords: Integrated Services, HIV, Tuberculosis (TB), Direct Observed Therapy (DOT), Antiretroviral (ARV), Community Health Workers

\section{Introduction}

HIV/AIDS continues to be a major public health issue with a global prevalence of $0.8 \%$ among adults [1]. An estimated
36.7 million people including 8 million children are currently living with the virus with just about $60 \%$ knowing they have the disease. Since the first case was diagnosed in 1987, the virus has claimed an estimated 35 million lives and in 2016, 1 million people were reported to have died from AIDS- 
related illnesses [2].

Tuberculosis (TB) is ranked as the ninth leading cause of death worldwide with a high co-infection rate with HIV/AIDS. In 2016, over 300,000 people living with HIV died from TB-related deaths and as reported by UNAIDS in 2017 [2], it was responsible for 1-in-3 AIDS-related deaths. Globally, the vast majority of patients from these diseases live in the Low- and Medium-Income Countries (LMICs).

Nigeria has the second highest burden of HIV globally as well as being the fourth biggest TB epidemic country worldwide [2] while also being one of the 10 countries that contribute $80 \%$ of the global HIV-TB coinfection [3].

Low antiretroviral treatment coverage $(30 \%)$ contributes to the high rates of $\mathrm{HIV}$-associated $\mathrm{TB}$ in Nigeria since antiretroviral treatment dramatically reduces a person living with HIV's risk of TB [2]. Nigeria also falls behind when it comes to providing preventative TB treatment. It is advised that those living with HIV are enrolled on the TB drug Isoniazid for preventative therapy, yet in Nigeria only $29 \%$ of people living with HIV have access to this medicine [1]. Cases of TB are underreported in Nigeria, and the country accounts for $8 \%$ of the global gap between TB incident cases and notifications [3].

Literature is replete of the mutual relationship between HIV and TB epidemics with HIV grossly identified as a key driver of the TB epidemic. Thus, making effective management (prevention and treatment) a useful tool in effective TB control [4]

For improved treatment outcomes, decreased burden of clinic attendance and follow up for patients, a scheme of HIV-TB integration services was proposed and currently being implemented. These result to patients lacking the motivation to adhere to treatment regimens. According to a WHO Technical brief [5], it describes integrated health services as the basis of Primary health care in the 1980s thus making the idea of integrated health services not being new. The brief further defined integrated health services as "the organization and management of health services so that people get the care they need, when they need it, in ways that are user friendly, achieve the desired results and provide value for money". However, the psychological and sociological needs of patients accessing HIV/TB integrated activities are ignored. There is the need to view the provision of HIV/TB integrated care from the patient's standpoint in order to achieve better treatment outcomes and to ensure that the needs of these patients are really being met [6]. This gap is compounded by the existence of very few published studies that assessed the efforts of integrating TB/HIV services in meeting HIV positive patient's needs.

A Cochrane Database review of integrated health services in Low and Medium Income Countries (LMICs) concluded that few good studies of rigourous study design had being carried out but they considered majorly the service supply side and proposed that future studies must assess the client's views of integration strategies as this infleunce uptake and effectiveness. [7] There is therefore the need to view the provision of HIV/TB integrated care from the patient's standpoint in order to achieve better treatment outcomes and to ensure that the needs of these patients are really being met. The rationale of this survey is to find out ways of enhancing the delivery of HIV/TB integrated services in order to improve adherence, treatment outcomes and the quality of life of through the perceptions of the patients.

\section{Method}

This is a descriptive cross-sectional survey implemented at the Lagos State University Teaching Hospital, Lagos among HIV positive clients enrolled on the HIV/TB integrated services in the hospital. A purposive sampling approach was used to select consecutive consenting clients on their clinic days. Trained data collectors were involved in this research work and questionnaires written in English language was used for the data collection. Data collected using semistructured questionnaires was post-code prior to entering into the SPSS version 21 software for analysis with data presentation in frequency and percentages. Ethical clearance was obtained from the Ethics Committee of the Texila American University Georgetown, Guyana, South America and the consent of the participants were sought before participation in the study.

\section{Result}

\subsection{Socio-demographic Characteristics of Respondents}

With reference to Table 1 below, the respondents of this survey are majorly female (80\% of HIV \& $60 \%$ of TB patients) with secondary education (about $40 \%$ for both cases). Over $50 \%$ of the respondents are married with $80 \%$ of the HIV patients and $65 \%$ of the TB patients being Christian.

Table 1. Socio-demographic characteristics of respondents.

\begin{tabular}{lll}
\hline \multirow{2}{*}{ Variables } & Adult HIV Positive $(\mathbf{n}=\mathbf{5 5})$ & TB $(\mathbf{n}=\mathbf{3 2})$ \\
\cline { 2 - 3 } & $\mathbf{n}(\mathbf{\%})$ & $\mathbf{n}(\mathbf{\%})$ \\
\hline Gender & & \\
Male & $11(20.0)$ & $13(40.6)$ \\
Female & $44(80.0)$ & $19(59.4)$ \\
Education & & \\
Uneducated & $5(9.1)$ & $9(28.1)$ \\
Primary & $10(18.2)$ & $14(43.8)$ \\
Secondary & $27(49.1)$ & \\
\hline
\end{tabular}




\begin{tabular}{lll}
\hline \multirow{2}{*}{ Variables } & Adult HIV Positive (n= 55) & TB (n= 32) \\
\cline { 2 - 3 } & $\mathbf{n}(\mathbf{\%})$ & $\mathbf{n}(\mathbf{\%})$ \\
\hline Graduate & $13(23.6)$ & \\
Occupation & & $1(3.1)$ \\
Student & $1(1.8)$ & $3(9.4)$ \\
Trader & $20(36.4)$ & \\
Artisan & $10(18.2)$ & $2(6.3)$ \\
Business & $3(5.5)$ & \\
Civil servant & $3(5.5)$ & \\
Professional (Accountant, Banker etc) & $1(1.8)$ & \\
Engineering & & $26(81.3)$ \\
Medical Practitioner & & \\
Driving & $1(1.8)$ & $7(21.9)$ \\
Not specified & $16(29.1)$ & $17(53.1)$ \\
Marital Status & & $8(25.0)$ \\
Single & $16(29.1)$ & \\
Married & $31(56.4)$ & \\
Divorced & $6(10.9)$ & $21(65.6)$ \\
Widow & $2(3.6)$ & $11(34.4)$ \\
Religion & & \\
Christianity & $44(80.0)$ & \\
Islam & $10(18.2)$ & \\
Traditional & $1(1.8)$ & \\
\hline & &
\end{tabular}

\subsection{Knowledge of Mode of Transmission of HIV by Respondents}

Table 2 below showed that majority of the respondents had good knowledge about the mode of transmission of HIV. Unprotected sexual intercourse as a mode of transmission was known the most ( $71 \%$ by HIV and $100 \%$ by TB patients) by the respondents which was followed by blood transfusion.

Table 2. Knowledge of respondents about mode of transmission.

\begin{tabular}{lll}
\hline & Adult HIV Positive $(\mathbf{n}=\mathbf{5 5})$ & TB $(\mathbf{n}=\mathbf{3 2})$ \\
\cline { 2 - 3 } & $\mathbf{n}(\mathbf{\%})$ & $\mathbf{n}(\mathbf{\%})$ \\
\hline Mode of spreading HIV & $38(71.7)$ & $32(100.0)$ \\
Unprotected Sexual Intercourse & $46(86.8)$ & $22(68.8)$ \\
Blood Transfusion & $33(62.3)$ & $23(71.9)$ \\
Unsterile Instrument & $28(52.8)$ & $13(40.6)$ \\
MTCT & $2(3.8)$ & \\
Others &
\end{tabular}

\subsection{Knowledge of Respondents on Antiretroviral}

In table 3 below, the ability of antiretroviral to prevent death from HIV/AIDS complications was largely seen as the importance of antiretroviral by over $70 \%$ of the respondents. Prevention of mother to child transmission by antiretroviral was known the least among respondents.

Table 3. Good knowledge of antiretroviral by respondents.

\begin{tabular}{lll}
\hline \multirow{2}{*}{ Antiretroviral } & Adult HIV Positive (n= 55) & TB (n= 32) \\
\cline { 2 - 3 } & $\mathbf{n}(\mathbf{\%})$ & $\mathbf{n}(\mathbf{\%})$ \\
\hline Drug to prevent transmission to baby & $11(22.9)$ & $1(3.2)$ \\
Drug to cure HIV & $19(39.6)$ & $1(3.2)$ \\
Drug to prevent death from HIV/AIDS & $36(75.0)$ & $27(71.1)$ \\
Drug to prolong lives & $28(58.3)$ & $9(23.7)$ \\
Works effectively with optimal adherence & $11(22.9)$ & \\
\hline
\end{tabular}

\subsection{Respondents' Reports on Accessing Integrated HIV-TB Services}

Table 4 as highlighted below revealed that majority (76\% of HIV and $97 \%$ of TB patients) reported to accessing treatment for $\mathrm{HIV}$ and $\mathrm{TB}$ in the same facility as well as being managed by the same nurses $(69 \%$ of HIIV patient and $94 \%$ of TB patients). However, for those accessing HIV services and TB services in separate facilities, the distance for the two services as reported by majority is between $5-10$ minutes. 
Table 4. Accessing integrated HIV/TB services care.

\begin{tabular}{|c|c|c|}
\hline & Adult HIV Positive $(n=55)$ & TB $(n=32)$ \\
\hline & $\mathbf{n}(\%)$ & $\mathbf{n}(\%)$ \\
\hline \multicolumn{3}{|c|}{ Receiving Treatment for both TB and HIV in same clinic or different clinic } \\
\hline Same & $42(76.4)$ & $31(96.9)$ \\
\hline Different & $13(23.6)$ & $1(3.1)$ \\
\hline \multicolumn{3}{|c|}{ Receiving Treatment for HIV diseases by same nurse or different nurses } \\
\hline Same & $38(69.1)$ & $30(93.8)$ \\
\hline Different & $17(30.9)$ & $2(6.2)$ \\
\hline 5 minutes to 10 minutes & $22(40.0)$ & $1(3.1)$ \\
\hline 10 minutes to 15 minutes & $3(5.5)$ & $1(3.1)$ \\
\hline 15 minutes to 20 minutes & & $1(3.1)$ \\
\hline 20 minutes to 25 minutes & $1(1.8)$ & $1(3.1)$ \\
\hline 25 minutes to 30 minutes & $5(9.1)$ & $1(3.1)$ \\
\hline Don't know & $47(43.6)$ & $27(84.4)$ \\
\hline
\end{tabular}

\subsection{Perception of Respondents About Health Workers Providing Integrated HIV-TB Services}

In table 5 below, it can be deduced that most ( $89 \%$ of HIV and $75 \%$ of TB patients) of the respondents in this survey responded "Yes" to the option of being visited by healthcare workers. However, over $60 \%$ of the respondents reported that they have never being visited by healthcare workers.

Table 5. Respondents 'perceptions of health workers in provision of integrated HIV-TB services.

\begin{tabular}{lll}
\hline & Adult HIV Positive (n= 22) & TB (n= 11) \\
\cline { 2 - 3 } & $\mathbf{n}(\mathbf{\%})$ & \\
\hline Would like to be visited by Health Care Workers & & \\
Yes & $49(89.1)$ & $24(75.0)$ \\
No & $6(10.9)$ & $8(25.0)$ \\
Ever Visited by Health Care Workers & & \\
Yes & $22(40.0)$ & $11(34.4)$ \\
No & $33(60.0)$ & $21(65.6)$ \\
Support received from Healthcare Worker & & \\
Health Education & $13(59.1)$ & $6(54.5)$ \\
Screen for TB & $1(4.5)$ & $4(36.4)$ \\
Voluntary counselling and HIV testing & $12(54.5)$ & $3(27.3)$ \\
Support for antiretroviral treatment & $17(77.3)$ & $11(100.0)$ \\
TB directly observed therapy (DOT) & $6(27.3)$ & $2(18.2)$ \\
Others & $2(9.1)$ & \\
\hline
\end{tabular}

\subsection{Advantages of Having Integrated HIV/TB Services in the Same Clinic}

According to table 6 below, the study reported that majority of the respondents cited "save cost of transportation" and "save time" as the major advantages of integrated HIV and TB services. Other notable advantages reported were treatment punctuality and patients' health status is known only to doctors and nurses in the same clinic.

Table 6. Reported advantages of integrated HIV/TB services.

\begin{tabular}{lll}
\hline \multirow{2}{*}{ Advantages of having services at same clinic } & Adult HIV Positive (n= 55) & TB $(\mathbf{n}=\mathbf{3 2})$ \\
\cline { 2 - 2 }$(\mathbf{n}(\%)$ & $\mathbf{n})$ \\
\hline Save cost of transportation & $45(81.8)$ & $14(43.8)$ \\
Treatment punctuality & $4(7.3)$ & $16(50.0)$ \\
Keeping to doctors' guideline and instruction & $0(0.0)$ & $1(3.1)$ \\
Remove worries and depression & $0(0.0)$ & $10(31.3)$ \\
Patients' Health status is known only to Doctors and Nurses in same clinic & $12(21.8)$ & $1(3.1)$ \\
It saves time & $28(50.9)$ & $18(56.3)$ \\
Enough time to see the Doctor & $0(0.0)$ & $7(21.9)$ \\
Not Indicated & $7(12.7)$ \\
\hline
\end{tabular}

\section{Discussion}

Our survey documented the perception of patients to the integrated HIV/TB services in a tertiary health facility in Lagos State, Nigeria as well as highlighted the advantages of effective integrated services of the disease-conditions under consideration.

Majority of the respondents were married, females with secondary education and predominantly Christians. This finding is in line with related studies that support the burden of the diseases under consideration to be higher among 
female gender [8, 9]. A study by Folajinmi, et al [10] in a survey of "determinant of optimal adherence to antiretroviral drugs among HIV positive clients in Federal Capital Territory of Nigeria" also emphasized the high prevalence of HIV among female Christians. This similarity could stem from the study area, being urban, which both studies were carried out and because of anecdotal notion that female Christians are allowed to access health services in health facilities alone much more than their Muslim counterparts.

The opinion of majority of respondents in this survey support visitation from health care workers even though when queried on reality of visitation by health workers, a majority of the respondents revealed that they haven't being visited. This emphasizes the limited number of health workers available in the health care system, as reported by closely related studies which outcomes also reported similar shortage of health workers in their surveys [11, 12, 13]. Allison et al [14] outcomes further support health care workers visitation to increase positive health outcomes as the neonatal mortality rate of 7.1 per 1000 live births was recorded among population studied for one year compared to the National value of 38 per 1000 live births while Tripathi et al [15] reported "quality evidence that home visits by trained community health workers are associated with improved care-seeking for ill young infants to health facilities in resource-limited settings".

The perception of our respondents about the services provided by health workers in HIV-TB integrated services is high for antiretroviral therapy support but relatively low for the Direct Observed Treatment (DOT) for TB which typified low knowledge of the integrated services among healthcare workers. Fairall et al. in their survey of "Effect of educational outreach to nurses in tuberculosis case detection and primary care of respiratory illness" noted the same challenge of poor knowledge and proffered that "To improve efficient HIV-TB services integration, the TB-HIV co-management courses should be included in the training courses for health workers so as to address poor health worker knowledge of the integrated services" [16]. Doherty et al also propose ongoing supportive supervision of healthcare workers at facilities for continuous delivery of quality care to clients [17].

The advantages of integrated HIV-TB services were reported by our respondents which align with previous related studies [18, 19], the similarity can be because of comparable low and medium income countries which these studies were carried out. However, our survey noted improved confidentiality of clients' medical history since "Patients' health status is known only to doctors and nurses in the same clinic". This survey established, as reported by the respondents, that the same healthcare workers are responsible for providing HIV and TB care services for them. They also reported that they access the integrated services in the same facility. This is in consonance Marian L and Virginia which advised that "Workers who previously looked after either patients with TB or patients with HIV should look after both, work under the same conditions for the same organization" [19]. This analogous opinion may be because both surveys considered the patients perception (demand side) of integrated services delivery.

\section{Conclusion}

There is a broad consensus on the value of integration of HIV-TB services in this region of the world where epidemic control of HIV and TB is still a challenge as the diseases are still marked with high mortalities among its patients. Effective integration is thought to increase access to and uptake of health services and to improve their efficiency and cost-effectiveness through better use of scarce resources especially as the global targets of making acute diseases (HIV, TB) a chronic disease by putting more sick individuals on their requisite medications - ART for HIV positive individuals and first or second line for TB; until suppression or cure is achieved. Our survey also contributes the demand side (patients) of the subject matter to the body of knowledge which is replete with literatures considering mostly the supply side (physicians) perspectives of integrated health services.

\section{Recommendation}

i. More health education should be carried out by healthcare professionals to HIV and TB clients accessing services at their facilities on the concept of HIV-TB integrated services.

ii. Adequate and sufficient training focusing on positive attitude towards clients accessing treatment at sites used for HIV-TB integrated services. Also, commensurate increase in remuneration for the expected increase in burden for health workers should be instituted.

iii. To curtail some of the disadvantages identified by this survey, massive roll-out of the concept of HIV-TB integrated services to all sites should be explored.

\section{References}

[1] UNAIDS (2016) 'HIV and AIDS estimates'.

[2] UNAIDS (2017) 'Ending AIDS: Progress towards the 90-9090 targets'.

[3] WHO (2017) 'Global Action Plan on HIV Drug Resistance 2017-2021'[pdf].

[4] Corbett E, Watt C, Walker N et al. (2003) The growing burden oftuberculosis: global trends and interactions with the HIVepidemic. Archives of Internal Medicine, 163, 10091021.

[5] WHO (2008) Integrated health services- what and why? Technical Brief No. 1.

[6] Sanou, A., Dembele, M., Theobald, S., Macq, J. (2004) Access and adhering to tuberculosis treatment: Barriers faced by patients and communities in Burkina Faso. Int J Tuberc Lung Dis 8: 1479-1483. 
[7] Briggs CJ, Garner P. (2006) Strategies for integrating primary health services in middle- and low-income countries at the point of delivery. Cochrane Database of Systematic Reviews, Issue 2. Art. No.: CD003318. DOI: 10.1002/14651858.CD003318.pub2.

[8] UNAIDS (2010). Report on the Global AIDS Epidemic. Geneva: UNAIDS.

[9] WHO (2016) 'WHO HIV Drug Resistance Report 2012' [pdf].

[10] Folajinmi O. Adebimpe O, Bodunde O, Daniel Ebenezer O and Mobolaji K (2018) Determinant of optimal adherence to antiretroviral drugs among HIV positive clients in Federal Capital Territory of Nigeria. International Journal for research in health sciences and nursing. Vol 5 issue 7.

[11] Breier M (2007) The Shortage of Medical Doctors in South Africa. Part of study: A multiple source identification and verification of scarce and critical skills in the South African labour market. Human Sciences Research Council.

[12] Wildschut A, Mqolozana T (2008) Shortage of Nurses in South Africa: Relative or Absolute? Part of study: A multiple source identification and verification of scarce and critical skills in the South African labour market: Human Sciences Research Council.

[13] Nansera D, Bajunirwe F, Kabakyenga J, Asiimwe PK, Mayanja-Kizza H (2010) Opportunities and barriers for implementation of integrated TB and HIV care in lower level health units: experiences from a rural western Ugandan district. Afr Health Sci 10: 312-319. [PMC free article] [PubMed] [Google Scholar].
[14] Allison Livingston, Angelo Tomedi, Alison Campbell, Carol Morales, and Mutuku A. Mwanthi. A. (2013) Community Health Worker Home Visitation Project to Prevent Neonatal Deaths in Kenya. Journal of Tropical Pediatrics, VOL. 59, NO. 1. doi: 10.1093/tropej/fms034.

[15] Tripathi A, Kabra SK, Sachdev HPS, Lodha R. (2016) Home visits by community health workers to improveidentification of serious illness and care seeking in newborns and young infants from low- and middle-income countries. Journal of Perinatology. 36, S73-S81. doi: 10.1038/jp.2016.34.

[16] Fairall L, Zwarenstein M, Bateman E et al. (2005) Effect of educational outreach to nurses on tuberculosis case detection and primary care of respiratory illness: pragmatic cluster randomized controlled trial. BMJ, 331, 750-754.

[17] Doherty T, Chopra M, Nsibande D \& Mngoma D (2009) Improving the coverage of the PMTCT programme through a participatory quality improvement intervention in South Africa. BMC Public Health, 9, 406.

[18] Legido-Quigley Helena, Catherine M. Montgomery, Palwasha Khan, Rifat Atun, Ade Fakoya, Haileyesus Getahun and Alison D. Grant (2013) Integrating tuberculosis and HIV services in low- and middle-income countries: a systematic review Tropical Medicine and International Health. Vol 18 No 2 pp 199-211.

[19] Marian L, Virginia Z. (2011) TB and HIV integration: obstacles and possible solutions to implementation in South Africa. Tropical Medicine and International Health. Vol 16 No 4 pp 431-438. 